\title{
Alpha correlates of practice during mental preparation for motor imagery
}

\author{
Mauro Nascimben, Yu-Kai Wang, Member IEEE, Jung-Tai King, Tzyy-Ping Jung, \\ Fellow IEEE, Jonathan Touryan, Member IEEE, Brent J. Lance, Senior Member \\ IEEE, Chin-Teng Lin, Fellow IEEE
}

\begin{abstract}
Performance of motor imagery (MI)-based brain-computer interface $(\mathrm{BCl})$ systems varies intraday and interday and practice is a fundamental way to stabilize effectiveness of outcomes over time. In this research, we recorded two experimental sessions from ten healthy subjects while playing a $\mathrm{BCl}$-oriented videogame for two weeks. Analysis focused on the exploration of electroencephalographic changes during mental preparation between novice and practiced subjects. EEG changes were quantified using global field power (GFP), dynamic time warping (TW) and mutual information (MutInf): GFP represents the strength of the electric field, TW measures signal similarities and Mutlnf signals interdependency. Significant results were in lower alpha for GFP and upper alpha for TW and Mutlnf. Findings suggest that EEG changes during mental preparation provide a quantitative measure of practice and mental effort. These metrics extracted before motor intention could be applied in $\mathrm{BCl}$ models targeting $\mathrm{MI}$ to monitor user training levels.

Index Terms-Brain Computer Interface, Motor Imagery, Mental Preparation, EEG
\end{abstract}

M. Nascimben is with Brain Research Center, National Chiao Tung University, Taiwan (e-mail: msnascimben@gmail.com).

Y. K. Wang is with Computational Intelligence and Brain Computer Interface Lab, Centre for Al, University of Technology Sydney, Australia (email: YuKai.wang@uts.edu.au)

J. T. King is with Brain Research Center, National Chiao Tung University, Taiwan (e-mail: jtchin2@gmail.com).

T. P. Jung is with Swartz Center for Computational Neuroscience, University of California, San Diego, USA (email: tpjung@ucsd.edu)

J. Touryan is with U.S. Army Research Laboratory, Aberdeen Proving Ground, MD, USA (email: jonathan.o.touryan.civ@mail.mil)

B. J. Lance is with U.S. Army Research Laboratory, Aberdeen Proving Ground, MD, USA (email: brent.j.lance.civ@mail.mil)

C. T. Lin is with Computational Intelligence and Brain Computer Interface Lab, Centre for Al, University of Technology Sydney, Australia (email: Chin-Teng.Lin@uts.edu.au)

This work was supported in part by the Australian Research Council (ARC) under discovery grant DP180100670 and DP180100656; NSW Defence Innovation Network and NSW State Government of Australia under the grant DINPP2019 S1-03/09; Office of Naval Research Global, US under Cooperative Agreement Number ONRG-NICOPN62909-19-1-2058. Research was also sponsored in part by the Army Research Laboratory and was accomplished under Cooperative Agreement Number W911NF-10-2-0022 and W911NF-10-D-0002/TO 0023.

\section{INTRODUCTION}

Brain-computer interfaces (BCIs) establish direct communication pathways between the human brain and the devices or systems to be controlled by interpreting the voluntary modulation of bio-signals. Most popular BCIs interpret encephalographic (EEG) brainwaves [1] as modulatory signal. One type of brainwave is the arciform or $\mathrm{mu}$ oscillation localized over central brain areas surrounding the primary motor cortex. A common class of BCIs encloses those based on changes in mu amplitude during imagined (voluntary) movements, known as motor imagery (MI)-based BCIs. When a person imagines a specific body movement (usually of their hands), a stereotypical attenuation of this mu rhythm, commonly referred to as an event-related desynchronization (ERD) in motor regions, can be observed [2]. This ERD modulation can then be converted to a command of the BCI system for the direct control of a device [3].

Despite the simplicity of this neurophysiological mechanism, the usage of MI-based BCIs is limited by variations in intraday and interday user performance [4]. Moreover, some users have been reported to be unable to control an MI-based BCI system using mu rhythm modulation [5]. Studies by Allison and Blankertz have shown that nearly $20-30 \%$ of subjects are unable to proficiently control MIbased BCIs [6], [7]. Previous studies have also suggested that high-performing BCI users are able to recruit MI-related brain networks, including supplementary motor areas, in a coordinated way [8], [9]. Likewise, attentional engagement, such as the ability to concentrate on a task, seems to play a role in MI performance outcomes [10], while anxiety has been associated with reduced executive performance [11], [12].

Practice can stabilize performance levels over time. A previous MI experiment using functional near-infrared spectroscopy showed an enhanced activation pattern due to practice [13]. Empirically, individuals report an elevated alertness to processing incoming stimuli, which may cause a higher use of resources than is necessary for an individual comfortable with the experimental environment. Users of MI BCIs show a similar activation pattern based on an awareness of task components and familiarity with the BCI system [14]. In addition, while performing an MI-based BCI task, motor programming, visual attention and temporal attention also play important roles. 


\section{ROLE OF MENTAL PREPARATION}

Extensive evidence has shown that there are many similarities between real movements and MI [15]. In fact, imagined motor movements preserve many properties such as the temporal sequence, programming rules, and neurophysiological aspects that are observed in the corresponding real actions [16], [17], [18]. Previous papers have reported that the cognitive processes underlying MI comprise two consecutive phases, mental preparation and movement imagination, for planning and performing a given task, respectively. [19], [20]. Our present interest is focused on finding preconscious EEG signatures of mental practice during mental preparation for MI. Mental practice of acted movements significantly increases the signal amplitudes and performance of the subsequent movement [21], [22]. Maeder et al. [23] formulated the hypothesis that mental preparation could affect MI performance. We build upon these results by exploring the effects of time and practice on mental preparation for MI. The proposed approach compared to previous literature includes frontal and parietal areas due to their involvement in memory and attentional circuits, analyzes induced changes not strictly related to power band modifications and identifies some metrics that could be easily collected as input features for online classification.

\section{EXPERIMENTAL HYPOTHESIS}

We assumed that the measured EEG signals would be related to the behavioral performance and attentional fluctuations of mental preparation for performance in the MIbased BCI. As a result, we explored the following hypotheses:

Hypothesis 1: Practice influences intersession alpha activity during the preparatory phase of MI. We explored this hypothesis by performing both intrasession and intersession comparisons of global field power (GFP).

Hypothesis 2: Practice increases the stability of the EEG signal amplitude during the preparatory phase of MI. We explored this hypothesis using time warping (TW) to compare both intrasession and intersession EEG signals. Empirically, an individual that becomes skilled in one task or action achieves maximum certainty on task execution with a minimum requirement of resources. In our case, the dynamics of resources allocated during mental preparation for MI should reflect the dynamics of strategic control of visuospatial attention. In complex visual environments with multiple spatially or temporally distributed sources of information, knowledge and experience on task should converge to an efficient management of incoming stimuli, allowing a disengagement of unnecessary attentional resources. Thus, the dynamics of resource allocation should be reflected in the neurophysiological signal as a function of attention allocation. For example, the amplitude of the N2pc component of eventrelated potentials reflects the level of attention on target stimuli. Similarly, the attention disengagement effect should be statistically interpretable through EEG properties found with TW as a direct consequence of mental practice on signal shape.
Hypothesis 3: Practice decreases interdependence between brain areas during the preparatory phase of motor activity. We explored this hypothesis by comparing mutual information (MutInf) across intrasession and intersession EEG signals. Recently, Bassett et al. [24] stated that with certain activities, such as playing an instrument, the more practiced the tasks, the less integrated and more autonomous motor and visual regions become. A similar specialization could be found during mental preparation through measures of Mutual Information. Specifically, in experienced subjects, we expected more independency between brain areas. In this case, specialization may be one of the steps needed to achieve efficient resource allocation.

In this paper, we explored the intrasession and intersession effects of time on task and practice on mu or alpha (which share the same frequency range) activity during the mental preparation phase of MI across a wide brain area, including frontal and parietal regions. We performed multichannel EEG recordings while participants played a videogame with an embedded MI-based BCI for two 90-minute sessions two weeks apart.

\section{METHODS}

\section{A. Participants}

Ten healthy right-handed male subjects aged 22-27 performed two 90-minute sessions two weeks apart (called novice and practiced sessions). This study was carried out in strict accordance with the recommendations in the Guide for Committee of Laboratory Care and Use of the National Chiao Tung University. Each participant read and signed an informed consent form before the experiment began.

\section{B. Experimental design}

The MI literature has suggested that a rich visual representation of the feedback signals (for example, in the form of a videogame) may enhance learning in BCI tasks [25], [26]. In addition, a videogame with engaging graphics stimulates participant attention and increases motivation. In our study, we used BCIGEM [27], which is a game-based scenario with five BCI paradigms. The BCIGEM videogame is a visual puzzle-matching game derived from a popular gemswap saga that is played for 2-3 minutes. At the end of each gem-swap round, participants were asked to perform the designed MI BCI paradigm. The sequence of gem-swap and MI task was repeated for 1.5 hours to collect multiple MI trials ("Game sequence" in Supplementary Materials). For this experiment, we configured BCIGEM for open-loop data collection with offline analysis. This study only analyzed brain activity correlated to mental preparation during BCIGEM MI task execution.

During the MI phase, participants had to imagine a hand movement related to the goal of the task. When a battery appeared in the center of the screen, subjects had to imagine a hand movement pushing the battery towards the robot on the 
left to boost their stamina. Likewise, when a bomb appeared in the center of the screen, an imagined right-handed movement was needed to throw it at the alien on the right side of the screen. Prior to this MI procedure, a preparatory video was shown where a crane at the top of the screen grabs the battery or the bomb, initiating the task. The time elapsing between the on-screen appearance and initial movement of the crane (to grab the battery or the bomb) was one second. We considered this phase to be the mental preparation for MI because participants needed to recognize the battery or the bomb and prepare their strategy for completing this stage of the game ("Epoch Description" in Suppl. Mat.). Throughout gameplay, EEG recordings were collected with a $32 \mathrm{AgCl}$ electrode cap (10-20 electrode position system) and acquired with a Neuro Scan NuAmps ${ }^{\mathrm{TM}}$ device. The sampling rate was set to $500 \mathrm{~Hz}$ with contact impedance below $5 \mathrm{k} \Omega$ and the extracephalic reference electrode placed on the mastoids. The experiment was carried out in a soundproof room. In the interval between the two experimental sessions (novice and practiced), the same subjects practiced the BCI videogame for one hour every day for two weeks.

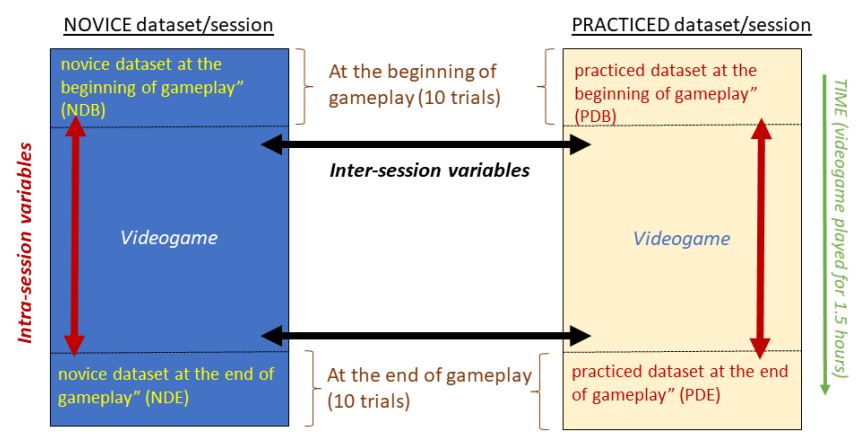

Fig. 1. Experimental schema: two datasets were recorded (BCI videogame with novice subjects and BCI videogame with the same subjects after practice).

\section{Pre-processing}

For compatibility with previous studies, we used a mental preparation window of $1000 \mathrm{~ms}$ before MI like in [23]. The mental preparation epoch was selected at time 0 from the onscreen appearance to $+1 \mathrm{~s}$ when the crane grabbed the battery or the bomb. The voltage of the EEG traces was normalized between 0 and 1 (feature scaling) and averaged across 10 epochs of each experimental condition. All recordings were visually inspected to remove artifacts, and traces were rereferenced using the common average mode. Lastly, brain potentials were filtered with a two-way least-squares finite impulse response (FIR) in alpha sub-bands. Filter order was calculated according to sampling frequency and bandwidth. Preprocessing resulted in four files, each representing an experimental condition: 'novice dataset at the beginning of gameplay' (i.e., NDB), 'novice dataset at the end of gameplay' (i.e., NDE), 'practiced dataset at the beginning of gameplay' (i.e., PDB), 'practiced dataset at the end of gameplay' (i.e., PDE) (Fig. 1).

In the current study, we divided the $8-12 \mathrm{~Hz}$ range of the mu rhythm [28] into two sub-bands of 8-10 Hz (low alpha/mu) and $10-12 \mathrm{~Hz}$ (high alpha/mu). For the rest of this paper, the terms 'upper alpha/mu' and 'lower alpha/mu' will be abbreviated to 'lower alpha' and 'upper alpha', meaning the sub-bands $8-10 \mathrm{~Hz}$ and 10-12 Hz, respectively.

\section{Statistical analysis}

MATLAB, including the EEGLAB toolbox [29], and SPSS software were used for the analysis. Statistical tests were performed with a significance level of $\alpha=0.05$. KolmogorovSmirnov Z test (K.S.), Mann-Whitney U (M.W. U) and Wilcoxon signed-rank (W) were mainly applied for the statistical tests ("Note on Statistical tests" in Suppl. Mat.). Nonparametric tests were selected because they are distribution free. When assumptions of common parametric tests, such as normality distribution or sphericity in ANOVA, were met, parametric tests were also included.

\section{E. Practice evaluation}

To measure the overall performance, we prepared an MIspecific assignment before the participants played the BCIGEM videogame. Subjects were instructed to imagine a brief hand movement (epoch length of $4 \mathrm{~s}$ considering one second of baseline) in the direction of an arrow shown on the screen (at $0 \mathrm{~s}$ time, 10 trials of MI right/10 trials of MI left). One cross was displayed at the center of the screen as an attentional cue $1 \mathrm{~s}$ before the arrow appeared. The time between two continuous MI trials was randomized. Each trial was analyzed in the $8-30 \mathrm{~Hz}$ band over 21 electrodes. A spatial filter was designed according to the Common Spatial Pattern (CSP) algorithm [30] and applied to the time series to determine the left and right MI movements in combination with the linear discriminant analysis (LDA) classifier [31]. The percentage of error of imagined movements (correct left or right minus imagined left or right)/correct left or right, declined between the two datasets: novice mean left/right: $35.3 \pm 2.3 \%$, practiced mean left/right: $27.6 \pm 0.7 \%$. A twosample t-test showed the significance of this difference (diff=7.7 $\mathrm{SE}=0.76 \mathrm{p}<0.001$ ). As intended, this preliminary evaluation proved that practice affected the MI ability of participants. A lower number of trials could explain some small differences in accuracy results from literature findings for naïve MI users [32].

\section{F. GFP}

To address Hypothesis 1, we explored the neural response strength using GFP [33], which is a reference independent measure that results in a waveform expressed in $\mu \mathrm{V}$ as a function of time. GFP can be calculated for each time point and provide instantaneous information on EEG potentials of an electrode montage. A high GFP can be interpreted as a modulation of synchronously activated generators across experimental conditions.

An analysis was conducted to investigate GFP in different brain regions under the four experimental conditions. To evaluate not only the primary motor areas but also regions surrounding the primary motor cortex, 15 electrodes $(\mathrm{F} 3, \mathrm{Fz}$, 
F4, FC3, FCz, FC4, C3, Cz, C4, CP3, CP4, CPz, P3, P4, Pz) were included in the analysis and grouped in five areas of interest:

- Frontal (F): F3, Fz and F4;

- Fronto-Central (FC): FC3, FCz, and FC4;

- Central (C): C3, Cz, and C4;

- Centro-parietal (CP): CP3, CPz, and CP4;

- Parietal (P): P3, Pz, and P4.

Frontal and Parietal electrodes were also added for their role in GO/NOGO tasks $[34,35]$ when subjects plan a motor action or they inhibit it. The GFP resulted in a matrix of locations (5) $\mathrm{x}$ conditions (4) $\mathrm{x}$ time. The length of the temporal epoch for GFP calculation could be the whole mental preparation epoch (1 s) or subintervals of it (200 or $100 \mathrm{~ms})$. Due to the additional property of GFP, subintervals of the whole epoch can be used to increase value density without introducing distortions in results.

\section{G. Dynamic $T W$}

To address Hypothesis 2, we compared the temporal characteristics of the EEG signals in every channel using dynamic TW. The usual method for comparing the evolution of data values over time is cross-correlation. In our case, we preferred a moment-by-moment measure of signal similarity, as the evolution of relevant neural signals during practice could imply that the same response would be elicited at different time points. Therefore, we used the dynamic TW algorithm to estimate dynamic correlations during the time course of the experiment and preserve the signal affinity between conditions. Dynamic TW was calculated in both the lower and upper alpha/mu intrasession sub-bands for each of the 15 electrodes. TW was computed by normalizing the length of the optimal warping path method: TW decreases when signals are similar.

\section{H. Mutual Information}

To investigate Hypothesis 3, we examined MutInf as a medium to compare the statistical properties of EEG signals. The normalized MutInf values of the fifteen electrodes were scored.

MutInf compares the statistical properties of two signals and quantifies the amount of information shared between them. MutInf is a dimensionless quantity: the higher the MutInf between two signals, the more information they contain and the more likely it is that the two signals are related.

\section{RESULTS}

Instead of an active process of imagined movement, motor preparation for MI represents the activation of neural circuits associated with premovement changes or planning. We divided the following Results section into three sub-sections evaluating the outcome of each statistical descriptor.

\section{A. $G F P$}

To evaluate Hypothesis 1, the whole-epoch GFP was analyzed (descriptive statistics are shown in Tables 1 and 2) using the $\mathrm{W}$ test to identify both intrasession and intersession macroscopic regional scalp differences. We found significantly lower intrasession alpha differences in both the novice and practiced datasets (M.W. U novice: $Z=-2.023$ $\mathrm{p}<0.05$, practiced: $Z=-2.402 \mathrm{p}<0.05)$. A decrease in GFP was found after practicing.

Table 1

GFP values in lower alpha (whole epoch)

\begin{tabular}{lllll}
\hline & NDB & NDE & PDB & PDE \\
\hline F & 101.60 & 100.79 & 107.01 & 80.94 \\
FC & 111.52 & 83.48 & 112.77 & 80.35 \\
$\mathrm{C}$ & 156.40 & 92.12 & 95.46 & 81.41 \\
$\mathrm{CP}$ & 193.72 & 154.11 & 145.19 & 103.10 \\
$\mathrm{P}$ & 179.42 & 175.37 & 133.20 & 88.67 \\
\hline Mean & 148.53 & 121.18 & 118.73 & 86.89 \\
\hline Std & 40.71 & 40.93 & 20.14 & 9.67 \\
\hline Median & 156.40 & 100.79 & 112.77 & 81.41 \\
\hline
\end{tabular}

Table 2

GFP values in upper alpha (whole epoch)

\begin{tabular}{lllll}
\hline & NDB & NDE & PDB & PDE \\
\hline $\mathrm{F}$ & 79.70 & 89.57 & 115.53 & 92.61 \\
$\mathrm{FC}$ & 74.89 & 77.98 & 107.19 & 100.98 \\
$\mathrm{C}$ & 128.62 & 99.84 & 103.51 & 98.03 \\
$\mathrm{CP}$ & 133.01 & 155.62 & 150.15 & 131.32 \\
$\mathrm{P}$ & 107.20 & 184.01 & 150.94 & 161.42 \\
\hline Mean & 104.68 & 121.41 & 125.46 & 116.87 \\
\hline Std & 26.89 & 45.97 & 23.30 & 29.11 \\
\hline Median & 107.20 & 99.84 & 115.53 & 100.98
\end{tabular}

The following analysis was centered on the lower alpha frequencies due to the significant results of GFP changes in this frequency range. For lower alpha activation, envelopes of maximal GFP values revealed that GFP decayed over the time course of whole experiment. This behavior is summarized in the boxplot diagrams of Fig. 2 .

Fig. 2. Boxplot of maximal GFP values (y-axis) over experiment time course in lower alpha. The four experimental conditions are on the $\mathrm{X}$-axis.

These GFP results showed that practice decreased GFP in lower alpha across sessions. To explore the GFP variations in more detail, values were recomputed and analyzed in time blocks of $100 \mathrm{~ms}$ and tested for normality with the ShapiroWilk test. Not all time sequences were normally distributed; 
therefore, we used the M.W. U test. The novice dataset (NDBNDE) and practiced dataset (PDB-PDE) showed significant intrasession variations (novice: $Z=-2.502 \mathrm{p}=0.012$, practiced: $\mathrm{Z}=-3.254 \mathrm{p}=0.001$ ). We explored the changes in the human brain during continuous performance of an MI-based BCI task considering practice and time on task as two separate phenomena. The intrasession difference in GFP could be related to a time-on-task phenomenon related to the mental fatigue associated with playing the videogame for one hour and half. However, M.W. U showed also significant intersession variations between the novice and practiced datasets at the beginning (NDB-PDB) of video gameplay $(\mathrm{Z}=-$ 2.558, $\mathrm{p}=0.011$ ). In this case, the difference between NDB and PDB evaluates the practice effect excluding mental fatigue.

Altogether, these variations can be conceptualized by the probability density function (PDF; Fig. 3) that portrays a scenario in which GFP peak values decrease during the experiment. Here, each PDF was created by summing the smoothed individual probability density curve for each data value every $100 \mathrm{~ms}$. In this case, not normalized values are displayed. Fig. 4 summarizes variations in GFP, showing that GFP values were negatively correlated with performance on the MI-based BCI task. The probability distribution of GFP amplitude became higher and narrower, while the mean of the PDF decreased as a consequence of MI-based BCI usage.

Fig. 3. Probability density functions describing GFP values (x axis) in lower alpha across the four experimental conditions. Note that the mean and standard deviation of the PDF both decrease with increased practice.

Fig. 4. The GFP over brain regions during mental preparation. Significant differences marked with '*'
Considering all brain areas, lower alpha GFP values mainly decay in central and posterior regions, suggesting a possible connection with parietal areas elaborating attentional processes (Fig. 4) related to mental preparation. A previous study on well-trained athletes stated that their optimal performance, compared to non-athletes, involves lower cortical activation in alpha sub-bands over centro-parietal sites [36], similarly to our GFP regional outcomes.

Regarding Hypothesis 1, we confirmed that practice affects intersession variables of mental preparation, while intrasession effects could be more easily explained with a time-on-task effect or mental fatigue. This isn't in contrast with previous literature because EEG alpha band shares modifications for different kind of mental activities [37] ranging from 'idling' to active cognitive processes, including motor training [38] and mental fatigue [39].

\section{B. Dynamic TW and MutInf}

TW and MutInf analysis techniques were applied on the same 15 scalp electrodes used for the GFP studies. Further analysis was mainly carried out between intersession changes on the same electrode set. Features between the novice and practiced datasets averaged over electrodes are shown as the mean and standard deviation in $200 \mathrm{~ms}$ time blocks (low alpha in Table 3 and high alpha in Table 4 with single electrode data available in Suppl. Mat.). The normality of the distribution was tested using the Shapiro-Wilk test, showing a normal distribution in only one of the five time sequences (practiced dataset TW: $\mathrm{W}=0.876, \mathrm{p}<0.001, \mathrm{df}=75$ ). Therefore, we applied nonparametric statistical calculations such as the M.W. U test. A significant difference during all epochs was found between the novice and practiced dataset in upper alpha band TW values $(Z=-5.268, p<0.000)$ and MutInf $(Z=-3.035$, $\mathrm{p}=0.002$ ). In lower alpha, normality was found for TW values in both datasets (novice: $\mathrm{W}=0.931, \mathrm{p}=0.001, \mathrm{df}=75$, practiced: $\mathrm{W}=0.905, \mathrm{p}<0.001, \mathrm{df}=75)$, and a two-sample t-test was applied (Levene test for equality of variances $\mathrm{F}=1.594$, $\mathrm{p}>0.05, \quad \mathrm{t}(148)=2.226, \quad \mathrm{p}=0.028)$, while the nonnormal distribution of MutInf suggested that M.W. U should be applied again $(Z=-1.366, p>0.05)$. Here, the TW results were significant in both sub-bands, but the MutInf in the lower alpha band was not. These findings suggest that practice influences similarity in both alpha sub-bands and dependency in the upper alpha signals over motor programming and visuoattentional-related cerebral areas. According to Hypothesis 2, practice stabilized the similarity between the PDB and PDE signals, thus decreasing the TW values between the novice and practiced datasets.

Table 3

Lower alpha: dynamic TW and MutInf averaged over electrodes

\begin{tabular}{l|ll|ll}
\hline \multicolumn{1}{l}{ Time } & \multicolumn{2}{c}{ TW } & \multicolumn{2}{c}{ MutInf } \\
& Novice & Practiced & Novice & Practiced \\
\hline $0-200 \mathrm{~ms}$ & $0.029 \pm 0.022$ & $0.027 \pm 0.014$ & $4.029 \pm 0.322$ & $3.937 \pm 0.292$ \\
$200-400 \mathrm{~ms}$ & $0.030 \pm 0.022$ & $0.024 \pm 0.018$ & $4.046 \pm 0.235$ & $4.054 \pm 0.359$ \\
$400-600 \mathrm{~ms}$ & $0.047 \pm 0.025$ & $0.023 \pm 0.016$ & $3.968 \pm 0.265$ & $4.125 \pm 0.258$ \\
$600-800 \mathrm{~ms}$ & $0.036 \pm 0.016$ & $0.033 \pm 0.019$ & $4.066 \pm 0.196$ & $4.160 \pm 0.365$
\end{tabular}


\begin{tabular}{l|ll|ll}
$800-1000 \mathrm{~ms}$ & $0.020 \pm 0.011$ & $0.017 \pm 0.021$ & $3.926 \pm 0.322$ & $4.062 \pm 0.267$
\end{tabular}

Table 4

Upper alpha: dynamic TW and MutInf averaged over electrodes

\begin{tabular}{l|ll|ll}
\hline \multicolumn{1}{l}{ Time } & \multicolumn{2}{c}{ TW } & \multicolumn{2}{c}{ MutInf } \\
& Novice & Practiced & Novice & Practiced \\
\hline $0-200 \mathrm{~ms}$ & $0.036 \pm 0.017$ & $0.018 \pm 0.011$ & $4.304 \pm 0.207$ & $4.173 \pm 0.293$ \\
$200-400 \mathrm{~ms}$ & $0.044 \pm 0.017$ & $0.028 \pm 0.018$ & $4.276 \pm 0.300$ & $4.103 \pm 0.321$ \\
$400-600 \mathrm{~ms}$ & $0.030 \pm 0.013$ & $0.025 \pm 0.014$ & $4.173 \pm 0.279$ & $4.170 \pm 0.216$ \\
$600-800 \mathrm{~ms}$ & $0.028 \pm 0.013$ & $0.022 \pm 0.024$ & $4.254 \pm 0.335$ & $4.126 \pm 0.211$ \\
$800-1000 \mathrm{~ms}$ & $0.037 \pm 0.012$ & $0.014 \pm 0.016$ & $4.287 \pm 0.272$ & $4.033 \pm 0.305$
\end{tabular}

Regarding Hypothesis 3, the findings in the upper alpha subbans suggest an increased independency with more evident changes in PDB-PDE than in NDB-NDE. To build a bivariate model for jointly portraying TW and the simultaneous MutInf changes evoked by mental practice, we focused on the upper alpha band, where both TW and MutInf changes were statistically significant. In the upper alpha band, changes during motor programming for imagined movement were restricted to the $\mathrm{FC} 3, \mathrm{FC} 4, \mathrm{FCz}, \mathrm{C} 3, \mathrm{C} 4, \mathrm{Cz}, \mathrm{CP} 3, \mathrm{CP} 4$, and $\mathrm{CPz}$ electrodes with values extracted every $200 \mathrm{~ms}$ during each epoch. These electrodes cover an area strictly surrounding the primary motor cortex. Table 5 presents descriptive statistics of the TW and MutInf data used in the following statistical analysis.

Table 5

Descriptive statistics of TW and MutInf over 9 channels

\begin{tabular}{lcc}
\hline $\begin{array}{l}\text { Upper } \\
\text { alpha }\end{array}$ & TW (mean \pm st dev) & MutInf (mean \pm st dev) \\
\hline $\begin{array}{l}\text { Novice } \\
\text { dataset }\end{array}$ & $0.034 \pm 0.015$ & $4.236 \pm 0.267$ \\
\hline $\begin{array}{l}\text { Practiced } \\
\text { dataset }\end{array}$ & $0.022 \pm 0.017$ & $4.063 \pm 0.269$ \\
\hline
\end{tabular}

The $\mathrm{W}$ test on the selected subset of electrodes in upper alpha was significant $(\mathrm{TW} Z=-3.138 \mathrm{p}=0.002$, MutInf $\mathrm{Z}=-$ $3.121 \mathrm{p}=0.002$ ), as were other nonparametric tests (TW: K.S. $Z=1.897, p=0.001$; M.W. U Z $=-3.7, p<0.000$; MutInf: K.S. $\mathrm{Z}=1.897, \mathrm{p}=0.001$; M.W. $\mathrm{U} \mathrm{Z}=-2.954, \mathrm{p}=0.003$ ). The linear relationship between corresponding pairs of TW and MutInf values was significant in both the novice $(\mathrm{F}=107.6882$ $\mathrm{p}<0.000)$ and practiced $(\mathrm{F}=39.2101 \mathrm{p}<0.000)$ datasets. Both datasets successfully passed Box's test of equality of covariance matrices $(\mathrm{M}=0.7955, \mathrm{~F}(3)=0.7759, \mathrm{p}>0.05)$. For the bivariate analysis, we applied both the Hotelling $\mathrm{T}^{2}$ test (related $\mathrm{T}^{2}=19.2189, \quad \mathrm{~F}(2,43)=9.3911, \quad \mathrm{p}=0.0004$, independent $\left.\mathrm{T}^{2}=20.4224, \mathrm{~F}(2,87)=10.0951, \mathrm{p}=0.0001\right)$ and multivariate ANOVA $\left(X^{2}=18.1567 \mathrm{df}=2 \mathrm{p}=0.0001\right.$, lambda $=$ 0.8116 , with homogeneity of variances confirmed by Levene test). Our statistical findings suggest that experience jointly influenced similarity and dependence in motor-related areas in upper alpha (Fig. 5).

Confirming our hypothesis, we found that the TW results were easily related to practice, which should cause increased similarity between signals highlighted by a TW decrease. However, for MutInf, an explanation of the practice effect was less obvious. As TW is unaffected by phase, we analyzed the phase of the signals as a possible explanation of increased independency in the practiced dataset with decreased values of MutInf. Instantaneous phase shifts inside the upper alpha band between the novice and practiced data sets were calculated in support of this idea by applying the Hilbert transformation on the signals from both datasets over $\mathrm{FC} 3, \mathrm{FCz}, \mathrm{FC} 4, \mathrm{C} 3, \mathrm{Cz}$, $\mathrm{C} 4, \mathrm{CP} 3, \mathrm{CP} 4$, and $\mathrm{CPz}$. The phase angle difference was extracted from the output of the Hilbert transformation. Phase differences were more sustained in the practiced datasets: $71 \%$ of the epoch points in the intersession signals of practice datasets had a higher instantaneous phase difference than those of novice datasets. These results are indicative of decreased interdependence between signals in the practiced condition.
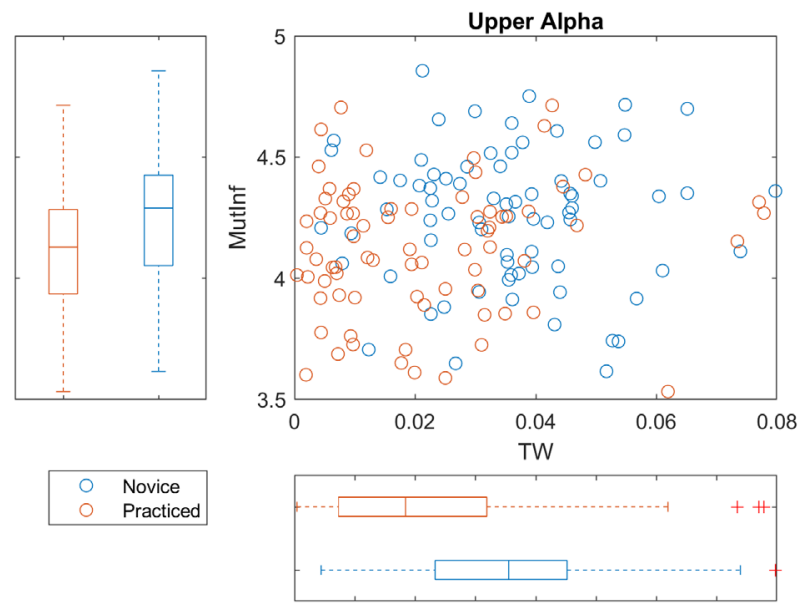

Fig 5. Single channel data of MutInf and TW during $1 \mathrm{~s}$ of mental preparation

\section{DISCUSSION}

The results show that our task manipulated induced changes in both lower and upper alpha/mu activities. The lower alpha/mu and upper alpha/mu are distinguished by their different roles in motor imagination [40]. Lower alpha/mu over central and parietal regions seems to be associated with general motor behavior, while upper alpha/mu desynchronizations are related to specific motor tasks [41]. In our work, we observed modulation in lower alpha/mu GFP, whereas the upper alpha/mu sub-band exhibited changes in both TW and MutInf before motor imagination ("Mathematical formulas" in Suppl. Mat.). Thus, we deduced that during mental preparation, we can identify two primary effects: 1) decreased GFP in lower alpha is positively correlated with the level of practice (intersession changes), and 2) upper alpha exhibits an inverse proportional relationship in TW-MutInf, with increased TW and decreased MutInf associated with practice.

"Intra-day" and "inter-day" variations are mainly a problem for BCI classifiers targeting MI because they reduce the performance of the BCI system. In the present paper we propose a way to extract three features from "mental preparation before MI" that could be useful in future works to compensate the "inter-day" and "intra-day" effect on BCIs 
working on MI. Features extracted from mental preparation could be used to determine the level of practice in the attempt to compensate "intra-day" (for example mental fatigue) or "inter-day" (for example changes in the ability to focus on the task due to psychological or physiological factors) variations. Authors of [4] provide a brief review of "intra-day" and "inter-day" factors affecting MI.

\section{A. Observed changes in brain activations induced by practice}

Previous studies have reported the roles of the lower and upper alpha bands not only in hand movements but also in attentional demands. Lower alpha has been associated with temporal attention when subjects prioritize the processing of certain task-relevant stimulus features to produce efficient behavior [42]. During the experiment, the appearance of the battery/bomb and the time taken by the crane to reach that object elicit the deployment of visual-attentional resources to specific brain areas that code the relevant feature of the scene [43] and [44], which enhances the speed or efficiency of the processes involved in the task. Intersession GFP decay could be related to this attentional process. For example, initially subjects pay attentional efforts to learn the structure of the videogame. The decrease in attentional resources deployed for learning results in reduced synchronization in the lower alpha band. In fact, the decreased GFP could be the consequence of a desynchronization in lower alpha. As shown in Fig. 4, we also found a more relevant effect in lower alpha over CP areas, which is compatible with the observations in cortical areas in recent literature [45]. A double role of the temporal orientation of attention in visual and motor programming has already been shown [46], which fits our hypothesis on the interaction of these two roles during mental preparation. In lower alpha, temporal attention in preparation for a general motor behavior should be modulated by practice, as stated in Hypothesis 1, where we theorized that practice could influence intersession alpha activity during the MI preparatory phase. However, we also observed modulations in the alpha band (intrasession) that could be related to how attention is conditioned by mental fatigue during long gameplay. This outcome could be explained by the fact that attention and mental fatigue distribute the same correlates in alpha band [47].

The similarity of the signals across alpha bands was greater in the practiced than in the novice dataset, providing some information about the task-specific motor system. Theoretically, after practice, subjects are better able to handle the MI-based task during the experimental situation: their stress level is low, and they have a high level of performance without wasting cognitive resources. Efficiency is reached when areas become specialized in one duty, deactivating unnecessary zones and stabilizing their activation pattern. This observation is in accordance with Hypothesis 2, which proposed that practice increases EEG signal amplitude stability during the MI preparatory phase.
Fig. 6. Ideal 3D feature space collecting experimental results on mental preparation. The black ellipsoid encloses the 1.5 standard deviation values is the novice dataset, and the red ellipsoid encloses that in the practiced dataset.

However, the MutInf results found in upper alpha could reflect a different concept. The amount of information that is 'shared' or 'mutual' in a neural sequence is based on the concept of entropy. This degree of relatedness between signals can be interpreted as the quantity of information that survives after 90 minutes of BCI usage. Decreased values of MutInf mean increased independence in practiced dataset values

(Hypothesis 3: practice decreases interdependence between brain areas during the preparatory phase of motor activity).

Moreover, prominent MutInf changes between datasets in the left hemisphere could be explained by the role of this hemisphere in temporal attention rather than in spatial attention. In [48], hemispheric asymmetries were reported with preferential right and left parietal activation for spatial and temporal attention, respectively. Practicing motor gestures generates mechanisms of cortical plasticity [49], and asymmetries can also provide an index of these alterations for mental preparation. For example, in [50] authors stated the possibility of modifications in the distribution of visuospatial attention caused by training in professional athletes. Practice could enhance skill acquisition, leading to an increased lateralization of attentional networks with increased signal independency [24]. Fronto-parietal attentional networks related to orienting follow a bilateral dorsal pathway in the brain. Skill acquisition could change the activation of these routes with a hemispheric prevalence, leading to a lateralized specialization. Thus, MutInf could be another correlate of temporal attention, similar to our hypothesis for GFP in lower alpha. We could link this observation with Hypotheses 2 and 3 , asserting that practice increases signal stability (shape of the evoked brainwaves or similarity obtained by TW) while simultaneously decreasing interdependence (reflected by signal phase changes through MutInf).

\section{B. Possible future application: practice level detection in $\mathrm{BCls}$}

Many studies have investigated the neural correlates of perceptual performance and enhancing cognitive performance in athletes by examining practice effects using classic psychological paradigms with reaction times, EEG or evoked neurophysiological signals in go/no-go tasks [51], [52], [53]. In our paradigm, we used a video game in the form of a BCI, and we propose ways in which our findings can be applied in 
this field. One potential future application could be developing a machine learning-based model for detecting the level of practice during the mental preparation phase in an MI task. To simulate this theoretical concept, we collected GFP values over five regions in the lower alpha and TW/MutInf over nine electrodes every $200 \mathrm{~ms}$ (adding Pz to provide a matrix of the same length as that for GFP). In this way, a 3D feature space representing mental practice could recognize the novicepracticed separation line (Fig. 6) and share data with the main MI algorithm about the subjective practice level. Mental preparation could be used as a secondary BCI providing feedback or supporting a main MI algorithm targeting sensory-motor rhythms. In this configuration a hybrid BCI including mental preparation could provide more evidence regarding the practice level reached by the MI BCI user. Moreover, during intra-session recordings, such hybrid BCI could monitor the mental state of the user and its task-related modifications. For example, in case of mental fatigue detection it could send a feedback signal to the user suggesting to take a break. Latter case could be achieved calculating GFP while combination of GFP together with TW and MutInf could be used for user practice level detection.

\section{CONCLUSION}

Mental preparation changes over time provide a quantitative measure of practice and mental effort via EEG recordings. Practicing an MI task involves motor-related areas surrounding the motor cortex and the attentional networks that connect these areas. We proposed that practice-induced changes in the alpha band during mental preparation could be schematized as an efficient resource allocation model.

This model processes the incoming inputs and manages assets in a manner that supports the brain's strategic goals, reducing energy losses and optimizing the time factor. Intersession decreases in GFP and increases in signal similarity may reflect this energy-saving mode. Conversely, decreases in dependency might indicate the increased specialization of task-related brain areas. These findings identify some brain signatures of the MI-based BCI learning effect in the mental preparation phase. In the future, these outcomes could be included in a broad framework of an hybrid BCI to quantify the level of experience gained and stabilize performance, enabling each user to have a training duration fitted to his or her neurophysiological parameters.

In this paper, we found that, under the theory of the allocation of resources, practice results in a decrease in lower alpha GFP associated with changes in attentional resources. We also observed changes in the upper alpha TW-MutInf bivariate relationship, which we interpret as a more direct practice effect on neural signal shape. The present study could be considered a pilot experiment for future investigations. We are also conscious of some limitations in this study, such as the number of participants and the absence of a control group. However, as a secondary BCI algorithm supporting a main $\mathrm{BCI}$ classifier targeting sensory-motor rhythms, we think that mental preparation could support and improve efficiency of the main MI BCI. With this article, we hope to open a discussion on the role of the proposed EEG variables over extended brain areas during mental preparation before MI.

\section{RefERENCES}

[1] Wolpaw, J. R., Birbaumer, N., McFarland, D. J., Pfurtscheller, G., \& Vaughan, T. M. (2002). Brain-computer interfaces for communication and control. Clinical neurophysiology, 113(6), 767-791.

[2] Pfurtscheller, G., \& Neuper, C. (1997). Motor imagery activates primary sensorimotor area in humans. Neuroscience Letters, 239(2), 65-68.

[3] Pfurtscheller, G., \& Da Silva, F. L. (1999). Event-related EEG/MEG synchronization and desynchronization: Basic principles. Clinical Neurophysiology, 110(11), 1842-1857.

[4] Ahn, Minkyu, and Sung Chan Jun. "Performance variation in motor imagery brain-computer interface: A brief review." Journal of neuroscience methods 243 (2015): 103-110.

[5] Blankertz, B., Sannelli, C., Halder, S., Hammer, E. M., Kübler, A., Müller, K. R., ... \& Dickhaus, T. (2010). Neurophysiological predictor of mu-based BCI performance. NeuroImage, 51(4), 1303-1309.

[6] Allison, B. Z., \& Neuper, C. (2010). Could anyone use a BCI? In D. S. Tan \& A. Nijholt (Eds.) Brain-Computer Interfaces: Applying our Minds to Human-Computer Interaction, pp. 35-54, London: Springer.

[7] Blankertz, B., Sanelli, C., Halder, S., Hammer, E., Kübler, A., Müller, K. R., ... \& Dickhaus, T. (2009). Predicting BCI performance to study BCI illiteracy. BMC Neuroscience, 10(Suppl 1), P84.

[8] Guillot, A., Collet, C., Nguyen, V. A., Malouin, F., Richards, C., \& Doyon, J. (2008). Functional neuroanatomical networks associated with expertise in motor imagery. NeuroImage, 41(4), 1471-1483.

[9] Halder, S., Agorastos, D., Veit, R., Hammer, E. M., Lee, S., Varkuti, B., ... $\&$ Kübler, A. (2011). Neural mechanisms of brain-computer interface control. NeuroImage, 55(4), 1779-1790.

[10] Hammer, E. M., Halder, S., Blankertz, B., Sannelli, C., Dickhaus, T., Kleih, S., ... \& Kübler, A. (2012). Psychological predictors of mu-BCI performance. Biological Psychology, 89(1), 80-86.

[11] Burde, W., \& Blankertz, B. (2006). Is the locus of control of reinforcement a predictor of brain-computer interface performance?. In Proceedings of the 3rd International Brain-Computer Interface Workshop and Training Course, vol. 2006, 108-109

[12] Schupp HT, Junghöfer M, Weike AI, Hamm AO. Attention and emotion: An ERP analysis of facilitated emotional stimulus processing. Neuroreport: For Rapid Communication of Neuroscience Research. 2003;14(8):1107-1110. [13] Kaiser, V., Bauernfeind, G., Kreilinger, A., Kaufmann, T., Kübler, A., Neuper, C., \& Müller-Putz, G. R. (2014). Cortical effects of user training in a motor imagery based brain-computer interface measured by fNIRS and EEG. Neuroimage, 85, 432-444.

[14] Witte M, Kober SE, Ninaus M, Neuper C, Wood G. Control beliefs can predict the ability to up-regulate sensorimotor rhythm during neurofeedback training. Frontiers in human neuroscience. 2013; 7

[15] Kranczioch, C., Mathews, S., Dean, P. J., \& Sterr, A. (2009). On the equivalence of executed and imagined movements: evidence from lateralized motor and nonmotor potentials. Human Brain Mapping, 30(10), 3275-3286.

[16] Jeannerod, M. (1994). The representing brain: Neural correlates of motor intention and imagery. Behavioral and Brain Sciences, 17, 187-245.

[17] Decety, J., \& Jeannerod, M. (1995). L'imagerie mentale et son substrat neurologique. Revue Neurologique, 151, 474-479.

[18] Decety, J. (1996). Neural representations for action. Reviews in the Neurosciences, 7, 285-297.

[19] Cunnington, R., Windischberger, C., \& Moser, E. (2005). Premovement activity of the pre-supplementary motor area and the readiness for action: Studies of time-resolved event-related functional MRI. Human Movement Science, 24(5), 644-656.

[20] Thoenissen, D., Zilles, K., \& Toni, I. (2002). Differential involvement of parietal and precentral regions in movement preparation and motor intention. The Journal of Neuroscience, 22(20), 9024-9034.

[21] Biddle, S. J. (1985). Mental preparation, mental practice and strength tasks: a need for clarification. Journal of sports sciences, 3(1), 67-74.

[22] Landers, D. M. (1983). The effects of mental practice on motor skill learning and performance: A meta-analysis. Journal of sport psychology, 5(1).

[23] Maeder, C. L., Sannelli, C., Haufe, S., \& Blankertz, B. (2012). Prestimulus sensorimotor rhythms influence brain-computer interface classification performance. IEEE Transactions on Neural Systems and Rehabilitation Engineering, 20(5), 653-662. 
[24] Bassett, D. S., Yang, M., Wymbs, N. F., \& Grafton, S. T. (2015). Learning-induced autonomy of sensorimotor systems. Nature neuroscience, 18(5), 744-751.

[25] Pineda, J. A., Silverman, D. S., Vankov, A., \& Hestenes, J. (2003). Learning to control brain rhythms: Making a brain-computer interface possible. IEEE Transactions on Neural Systems and Rehabilitation Engineering, 11(2), 181-184.

[26] Lotte, F., Faller, J., Guger, C., Renard, Y., Pfurtscheller, G., Lécuyer, A., \& Leeb, R. (2012). Combining BCI with virtual reality: Towards new applications and improved BCI. In B. Z. Allison et al. (Eds.), Towards Practical Brain-Computer Interfaces, pp. 197-220, Berlin Heidelberg: Springer.

[27] Lance, B.J., Touryan, J., Wang, Y.K., Lu, S.W., Chuang, C.H., Khooshabeh, P., Sajda, P., Marathe, A., Jung, T.P., Lin, C.T. and McDowell, K. (2017) Towards serious games for improved BCI. Handbook of Digital Games and Entertainment Technologies, pp.197-224. Springer, Singapore

[28] Neuper, C., Scherer, R., Wriessnegger, S., \& Pfurtscheller, G. (2009). Motor imagery and action observation: Modulation of sensorimotor brain rhythms during mental control of a brain-computer interface. Clinical Neurophysiology, 120(2), 239-247.

[29] Delorme, A., \& Makeig, S. (2004). EEGLAB: An open source toolbox for analysis of single-trial EEG dynamics including independent component analysis. Journal of Neuroscience Methods, 134(1), 9-21.

[30] Ramoser, H., Muller-Gerking, J., \& Pfurtscheller, G. (2000). Optimal spatial filtering of single trial EEG during imagined hand movement. IEEE transactions on rehabilitation engineering, 8(4), 441-446.

[31] Wu, S. L., Wu, C. W., Pal, N. R., Chen, C. Y., Chen, S. A., \& Lin, C. T. (2013, April). Common spatial pattern and linear discriminant analysis for motor imagery classification. In Computational Intelligence, Cognitive Algorithms, Mind, and Brain (CCMB), 2013 IEEE Symposium on (pp. 146151)

[32] Blankertz, B., Sannelli, C., Halder, S., Hammer, E. M., Kübler, A., Müller, K. R., ... \& Dickhaus, T. (2010). Neurophysiological predictor of mubased BCI performance. NeuroImage, 51(4), 1303-1309.

[33] Skrandies, W. (1990). Global field power and topographic similarity. Brain Topography, 3(1), 137-141.

[34] Burle, B., Vidal, F., \& Bonnet, M. (2004). Electroencephalographic nogo potentials in a no-movement context: the case of motor imagery in humans. Neuroscience letters, 360(1-2), 77-80.

[35] van Boxtel, G. J., van der Molen, M. W., Jennings, J. R., \& Brunia, C. H. (2001). A psychophysiological analysis of inhibitory motor control in the stop-signal paradigm. Biological psychology, 58(3), 229-262.

[36] Del Percio, C., Babiloni, C., Bertollo, M., Marzano, N., Iacoboni, M., Infarinato, F., ... \& Comani, S. (2009). Visuo-attentional and sensorimotor alpha rhythms are related to visuo-motor performance in athletes. Human brain mapping, 30(11), 3527-3540.

[37] Baars, B. J., \& Gage, N. M. (2010). Cognition, brain, and consciousness: Introduction to cognitive neuroscience. Academic Press.

[38] Neuper, C., Schlögl, A., \& Pfurtscheller, G. (1999). Enhancement of leftright sensorimotor EEG differences during feedback-regulated motor imagery. Journal of Clinical Neurophysiology, 16(4), 373-382.

[39] Trejo, L. J., Kubitz, K., Rosipal, R., Kochavi, R. L., \& Montgomery, L. D. (2015). EEG-based estimation and classification of mental fatigue. Psychology, 6(05), 572.

[40] Pfurtscheller, G., Neuper, C., Flotzinger, D., \& Pregenzer, M. (1997). EEG-based discrimination between imagination of right and left hand movement. Electroencephalography and Clinical Neurophysiology, 103(6), $642-651$

[41] Pfurtscheller, G., Neuper, C., \& Krausz, G. (2000). Functional dissociation of lower and upper frequency mu rhythms in relation to voluntary limb movement. Clinical Neurophysiology, 111(10), 1873-1879.

[42] Babiloni, C., Miniussi, C., Babiloni, F., Carducci, F., Cincotti, F., Del Percio, C., ... \& Rossini, P. M. (2004). Sub-second "temporal attention" modulates alpha rhythms. A high-resolution EEG study. Cognitive Brain Research, 19(3), 259-268.

[43] Correa, Á., Lupiáñez, J., Madrid, E., \& Tudela, P. (2006). Temporal attention enhances early visual processing: A review and new evidence from event-related potentials. Brain Research, 1076(1), 116-128.

[44] Klaver, P., Smid, H. G., \& Heinze, H. J. (1999). Representations in human visual short-term memory: An event-related brain potential study. Neuroscience Letters, 268(2), 65-68.

[45] Agosta, S., Magnago, D., Tyler, S., Grossman, E., Galante, E., Ferraro, F., ... \& Battelli, L. (2017). The pivotal role of the right parietal lobe in temporal attention. Journal of cognitive neuroscience, 29(5), 805-815.
[46] Corbetta, M., \& Shulman, G. L. (2002). Control of goal-directed and stimulus-driven attention in the brain. Nature Reviews Neuroscience, 3(3), 201-215.

[47] Faber, L. G., Maurits, N. M., \& Lorist, M. M. (2012). Mental fatigue affects visual selective attention. PloS one, 7(10), e48073.

[48] Coull, J. T., \& Nobre, A. C. (1998). Where and when to pay attention: The neural systems for directing attention to spatial locations and to time intervals as revealed by both PET and fMRI. The Journal of Neuroscience, 18(18), 7426-7435.

[49] Schwenkreis, P., El Tom, S., Ragert, P., Pleger, B., Tegenthoff, M., \& Dinse, H. R. (2007). Assessment of sensorimotor cortical representation asymmetries and motor skills in violin players. European Journal of Neuroscience, 26(11), 3291-3302.

[50] Giglia, Giuseppe, et al. Visuospatial attention lateralization in volleyball players and in rowers. Perceptual and motor skills, 2011, 112.3: 915-925.

[51] Radlo, S. J., Janelle, C. M., Barba, D. A., \& Frehlich, S. G. (2001). Perceptual decision making for baseball pitch recognition: using P300 latency and amplitude to index attentional processing. Research quarterly for exercise and sport, 72(1), 22-31.

[52] Kida, N., Oda, S., \& Matsumura, M. (2005). Intensive baseball practice improves the Go/Nogo reaction time, but not the simple reaction time. Cognitive brain research, 22(2), 257-264.

[53] Nakamoto, H., \& Mori, S. (2012). Experts in fast-ball sports reduce anticipation timing cost by developing inhibitory control. Brain and cognition, $80(1), 23-32$ 